\title{
Oxalate Content of Egyptian Grown Fruits and Vegetables and Daily Common Herbs
}

\author{
Aly R. Abdel-Moemin ${ }^{1}$ \\ ${ }^{1}$ Nutrition and Food Science Department, Faculty of Home Economics, Helwan University, Cairo, Egypt \\ Correspondence: Aly R. Abdel-Moemin, Nutrition and Food Science Department, Faculty of Home Economics, \\ Helwan University, Cairo, Egypt. Tel: 202-3560-1762. E-mail: dralymoemin@yahoo.co.uk
}

Received: February 25, 2014 Accepted: March 13, 2014 Online Published: April 10, 2014

doi:10.5539/jfr.v3n3p66 URL: http://dx.doi.org/10.5539/jfr.v3n3p66

\begin{abstract}
Egyptian dieticians typically rely on foreign databases to find out oxalate content of food due to unavailability of local databases. The soil, fertilizers, climate and cultivars are often very different. Therefore, the purpose of this study is to establish a local database of oxalate content in Egyptian grown fruits and vegetables and selected daily common herbs. The current study analysed the total and the soluble oxalate in 37 Egyptian grown fruits, vegetables and 9 commonly used herbs. Two methods were used for screening the Egyptian foods for oxalate concentration; the first method was AOAC 1999 and the second was enzymatic method. Total oxalate varied greatly among the vegetables examined, ranging from 4 to $917 \mathrm{mg} / 100 \mathrm{~g} \mathrm{~F}$.W. Total oxalate of analysed fruits ranged from 9 to 50 $\mathrm{mg} / 100 \mathrm{~g} \mathrm{~F}$.W. There is a strong correlation found between the two methods used. Vegetables were classified into 4 categories; low oxalate concentration containing less than $10 \mathrm{mg}$ of oxalic acid $/ 100 \mathrm{~g} \mathrm{~F}$.W., such as cabbage, courgette, cucumbers, garlic, spring onions and turnip. Moderate oxalate concentration vegetables containing 10-25 mg/100 g F.W., such as aubergine, field bean, corn, peppers and watercress. High oxalate concentration vegetables containing $26-99 \mathrm{mg} / 100 \mathrm{~g}$ F.W., such as fūl, green beans, celery, mallow, okra and sweet potatoes. Very high oxalate concentration containing 100-900 mg/100g F.W. such as Swiss chard, molokhia, purslane and vine leaves (fresh). Extensive amounts of total oxalate (201-4014 mg/100 g D.W.) were found in daily common herbs such as caraway seed, green cardamom, cinnamon, coriander seeds, cumin, curry powder, ginger and turmeric powder.
\end{abstract}

Keywords: total oxalate, soluble oxalate, enzymatic method, fruits and vegetables, herbs

\section{Introduction}

Oxalic acid (ethanedioic acid), the name comes from the plant Oxalis (wood sorrel) from which it was first isolated (Liebman, 2002), occurs ubiquitously in nature, sometimes as a free acid, but more commonly as soluble potassium, sodium or ammonium oxalate or as insoluble calcium oxalate. Biosynthesis of oxalate occurs in members of all five kingdoms. Oxalate is associated with metabolic disorders and infectious diseases (Holmes \& Assimos, 1998; Nakagawa et al., 1999).

Oxalic acid and its salts occur as an end product of metabolism in a number of plants. When these plants are eaten they may have an adverse effect on humans and animals because oxalate binds calcium and other minerals and may cause stone formation in the urinary tract when the acid is excreted in the urine (Noonan \& Savage, 1999).

Oxalate-producing plants, which include many crop plants, accumulate oxalate in the range of $3 \%-80 \%(\mathrm{w} / \mathrm{w})$ of their dry weight (Libert \& Franceschi, 1987). The oxalate production pathways the cleavage of isocitrate, hydrolysis of oxaloacetate, glycolate/glyoxylate oxidation, and/or oxidative cleavage of L-ascorbic acid (Hodgkinson, 1977). Of these pathways, the cleavage of ascorbic acid appears to be the most common (Yang \& Loewus, 1975; Nuss \& Loewus, 1978; Li \& Franceschi, 1990; Keates et al., 2000).

The diversity of calcium oxalate crystal shapes and sizes, as well as their prevalence and spatial distribution, have led to a number of hypotheses regarding crystal function in plants. The proposed functions include roles in ion balance, in plant defense, in tissue support, in detoxification, and in light gathering and reflection (Franceschi \& Horner, 1980). Recently, (Nakata \& McConn, 2000) hypothesized the roles of calcium oxalate formed in plants in supporting tissue structure and in regulating excess tissue calcium. 


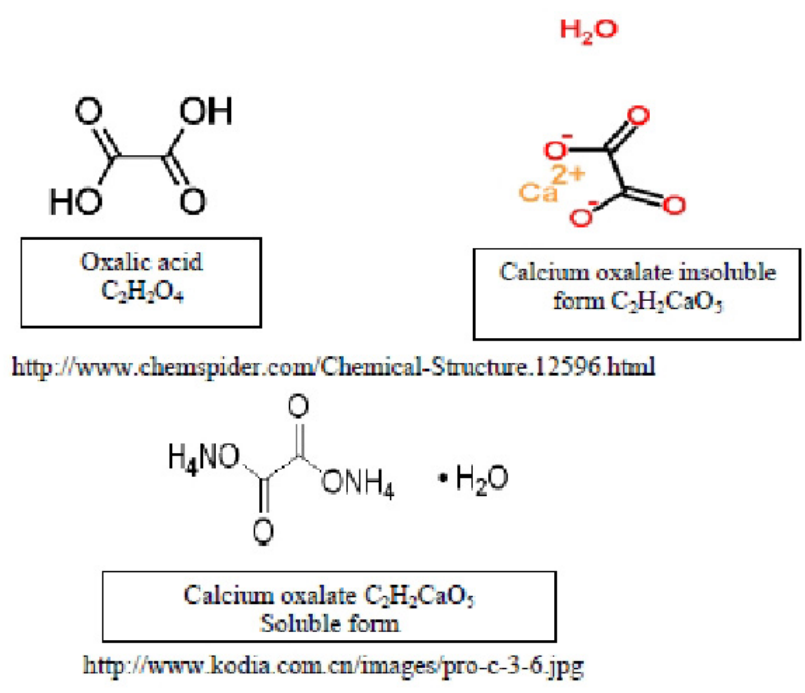

Figure 1. The chemical structure of different forms of oxalic acid

The oxalate content of food such as spinach can vary considerably between plants of the same species, due to differences in climate, soil quality, state of ripeness, or even which part of the plant is analyzed. Variations also may be caused by the different methods used for measuring oxalate in food (Eheart \& Massey, 1962).

Insoluble oxalate crystals formed in the gut are not absorbed and are carried out with the feces, thus reducing the bioavailability and absorption of calcium and iron in diets (Bataille \& Fournier, 2001). Human urine always contains small levels of calcium oxalate (Oke, 1969) that may be deposited in the kidneys of certain people as a common form of kidney stones (Massey et al., 1993).

In Egypt we have not enough data about how much oxalate in local grown fruits and vegetables or execrated oxalate in urine that comes from endogenous metabolic synthesis. On the other hand, healthy adults, with U.S. or European type western diets, $90 \%$ of oxalic acid excreted in the urine comes from endogenous metabolic synthesis (Massey et al., 1993). However, establishing a local database of oxalate content in Egyptian grown fruits and vegetables and daily common herbs may help Egyptian patients with hyperoxaluria to make more accurate dietary modifications.

\section{Materials and Methods}

\subsection{Experimental Procedure}

Thirty seven fruits and vegetables were analysed, the selection of which was based on those listed in the USDA Table 1984 with some modification to suit Egyptian food habits. The individual products were purchased from the same batch within the supermarket. On the day of purchase, where applicable, the edible parts of the fruits or vegetables were washed, dried, cut finely and weighed. For the purposes of this study three replicate samples of each product were used. In the case of products such as, cauliflower, and onions, the products were of a sufficient size and weight to allow for individual items to be used as single replicate. In the case of apricots, three individual fruits were taken as one replicate due to the small size, chopped up and a composite sample prepared from which the required sample weight was taken. Three replicates of each product were analysed in the dried state. Herbs were treated similarly apart from washing process. Following sampling and preparation as described above, three sub-samples of each ground product were prepared for the analysis. All results were expressed as mg oxalate/100 fresh weight (F.W.) of each fruit or vegetable or mg oxalate/100 dry weight (D.W.) for daily common herbs.

\subsection{Drying Food Samples}

A fruit or vegetable was dried at $28{ }^{\circ} \mathrm{C}$ from 2-7 days, depending on the type of food in a vacuum dryer (Remplissage evacuation, Arthermo Gessate MI, temperature and density guide, Italy). For example, spinach leaves and upper part of stalk of spinach were dried at $28^{\circ} \mathrm{C}$, for $3 \mathrm{~h}$ then broken into small pieces by hand, and mixed spinach and left in the vacuum dryer for about 27 hours until dried. Moisture and dry matter were determined in all foods (Infrared Moisture Determination Balance FD-610-Japan). 


\subsection{Optimizing Methods to Determine Oxalate Concentration in Foods}

Four methods were experimented to compare the accuracy and precision of oxalate methods in order to choose the best methods for analysing oxalate in Egyptian foods. These methods were Baker 1952, Oke 1969, AOAC 1999 and enzymatic method (kit). Spinach sample was used to optimize the methods in this study. The best results of the pilot study were obtained from the methods of AOAC 1999 and enzymatic method (Kit) presented in Table 1, compared to other methods.

\subsection{Determination of Oxalate by AOAC 1999}

According to the method of AOAC 1999 oxalate was determined; $0.1 \mathrm{~g}$ of food powder sample was weighed and mixed with $30 \mathrm{ml}$ of $1 \mathrm{M} \mathrm{HCl}$. Each mixture was then shaken in a water bath at $100^{\circ} \mathrm{C}$ for $30 \mathrm{~min}$. To each mixture was added $0.5 \mathrm{ml}$ of $5 \%$ calcium chloride and thoroughly mixed to precipitate out the calcium oxalate. The suspension was centrifuged at $800 \mathrm{~g}$ for 15 minutes and the supernatant poured out. The pellet was washed twice with $2 \mathrm{ml}$ of $0.35 \mathrm{M} \mathrm{NH} 4 \mathrm{OH}$ then dissolved in $0.5 \mathrm{M} \mathrm{H}_{2} \mathrm{SO}_{4}$. The solution was titrated with standard solution of $0.1 \mathrm{M} \mathrm{KMnO} 4$ with the temperature being maintained at $60^{\circ} \mathrm{C}$ to a faint violet colour that persisted for at least 15 seconds. For soluble oxalates, the same procedure was used except that instead of extracting the oxalates with 30 $\mathrm{ml}$ of $1 \mathrm{M} \mathrm{HCl}$, the extraction was with $30 \mathrm{ml}$ distilled water. The total and soluble oxalate contents were calculated as percent of D.W.

\subsection{Determination of Oxalate by Enzymatic Method (Kit)}

An enzymatic method (Trinity Biotech) proposed for the determination of oxalate in urine is adapted to estimate the total and soluble oxalate concentration in the examined foods. The method is based on the oxidation of oxalate by the oxidase enzyme and the determination of the resulting hydrogen peroxide, which in presence of peroxidase, 3-methyl-2 benzotiazinolone and 3-dimethylamino benzoic, gives an indamine compound with an absorption maximum at $590 \mathrm{~nm}$ as explained in Figure 2.
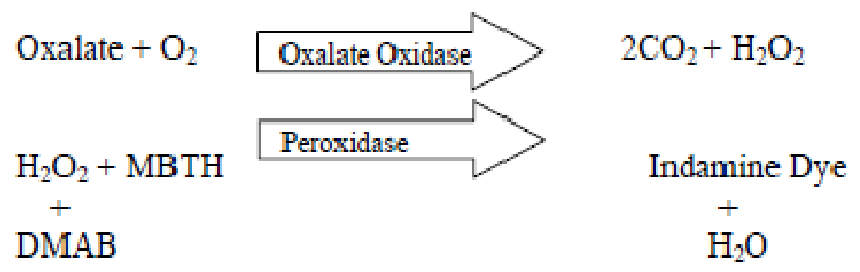

The principle of enzymatic method for oxalate determination.

The oxalate kit (Procedure No. 591; Trinity Biotech Plc Bray, Co. Wicklow, Ireland) was used to determine oxalic acid concentrations in the fresh grown produce of Egyptian fruits and vegetables and selected daily herbs as described by Palaniswamy et al. (2004) and Quinteros (2003) with little modification. A mass of $0.01 \mathrm{~g}$ food sample was homogenized in $5 \mathrm{~mL}$ deionized water (for soluble oxalate) or in $5 \mathrm{~mL} 2 \mathrm{~N} \mathrm{HCl}$ (for total oxalate) for 8 min with MPW-120 homogenizer at a maximum speed. The water soluble samples were diluted with $5 \mathrm{~mL}$ EDTA (10 mM, pH 7.6) (in order to chelate the calcium within the sample). Diluted samples were purified with sample purifying tubes which contained activated charcoal and centrifuged in sample-purifying tubes at 3,500 rpm for 5 min. The supernatant was collected via filtering through filter paper. Oxalate reagents were warmed to $37^{\circ} \mathrm{C}$; test tubes were labeled for blank, control, standard and sample; $1 \mathrm{~mL}$ oxalate reagent A 3-(dimethylamino benzoic acid) $(\mathrm{DMAB})+(3$-methyl-2-benzothiazolinone hydrazone) $\mathrm{MBTH}, \mathrm{pH}=3.1$ ) was added to each tube; $50 \mu \mathrm{L}$ of sample were added to each sample tube; $50 \mu \mathrm{L}$ deionized water was added to the blank and control tubes; $50 \mu \mathrm{L}$ oxalate standard were added to the standard tube; $0.1 \mathrm{~mL}$ of oxalate reagent $\mathrm{B}$ (oxalate oxidase and peroxide) was added to all tubes and immediately mixed by gentle inversion. All tubes were incubated at $37^{\circ} \mathrm{C}$ for $5 \mathrm{~min}$. The absorbance of blank, control, standard, and sample were determined at $590 \mathrm{~nm}$ in a CT-2200 Spectrophotometer, E-Chrom Tech, Taiwan. Measurements were taken twice to obtain consistent absorbances. Corrected absorbances were determined by subtracting the blank absorbance from absorbance readings of standard, control and the sample. The oxalate concentration in $\mathrm{mg}$ per $100 \mathrm{~g}$ of fresh weight was calculated on fresh fruits and vegetables and dry weight basis for herbs. 
Table 1. The amount of reagents used for blank the standard and the sample

\begin{tabular}{|c|c|c|c|}
\hline Control (N-E*) & Blank & Standard & Sample \\
\hline $1 \mathrm{~mL}$ Reagent $\mathrm{A}$ & $1 \mathrm{~mL}$ Reagent $\mathrm{A}$ & $1 \mathrm{~mL}$ Reagent $\mathrm{A}$ & $1 \mathrm{~mL}$ Reagent $\mathrm{A}$ \\
\hline & & & \\
\hline & & $50 \mu \mathrm{L}$ oxalate standard & - \\
\hline $0.1 \mathrm{~mL}$ Reagent $\mathrm{B}$ & $0.1 \mathrm{~mL}$ Reagent B & $0.1 \mathrm{~mL}$ Reagent B & $0.1 \mathrm{~mL}$ Reagent B \\
\hline
\end{tabular}

$* \mathrm{~N}=$ normal level-E elevated level.

\subsection{Statistical Analysis}

The results are presented as mean of three determinations \pm standard error $( \pm$ SEM). Pearson correlation coefficients ( $r$ ) were computed to assess the strength of the association between oxalate levels measured by AOAC 1999 and enzymatic methods. Oxalate values were analysed in transformed scale $\left(\log _{10}(\mathrm{X})\right.$ for simple linear regression Wessa (2012).

\section{Results}

The total and soluble oxalate content of the selected grown Egyptian fruits and vegetables and common herbs are presented in Table 2 to Table 8. However, the amount of insoluble oxalate can be evaluated as the difference between the total and the soluble oxalate (Rahman et al., 2008). It is noted that fruits and vegetables contain varying amounts of total oxalate. The outcome of Pearson correlation in this study was strong correlation that equal 1 between the two methods used for determination of oxalate in all foods examined. Linear regression analysis of the total oxalate concentration in vegetables revealed a linear correlation $\left(r^{2}=1.006\right)$ between the two methods (Figure 2). A similar correlation between the two methods occurred for the fruit samples $\left(\mathrm{r}^{2}=0.8739\right)$, (Figure 3) and $\left(r^{2}=0.9969\right)$ (Figure 4$)$ for herbs.

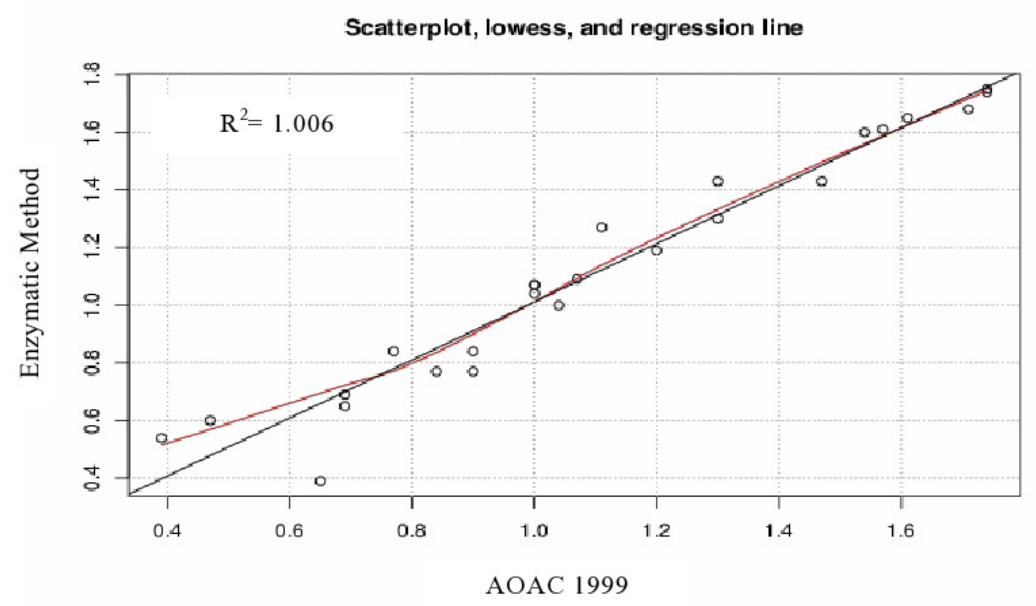

Figure 2. Simple linear regression analysis of the total oxalate between AOAC 1999 and enzymatic methods for vegetables; the values used were the log transformation 


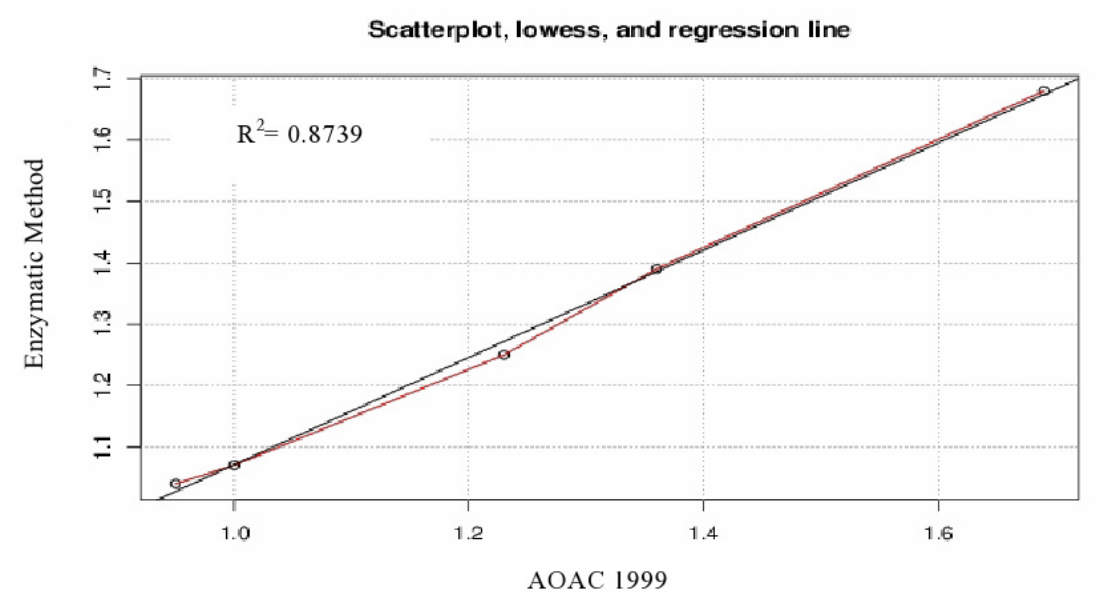

Figure 3. Simple linear regression analysis of the total oxalate between AOAC 1999 and enzymatic methods for fruits; the values used were the log transformation

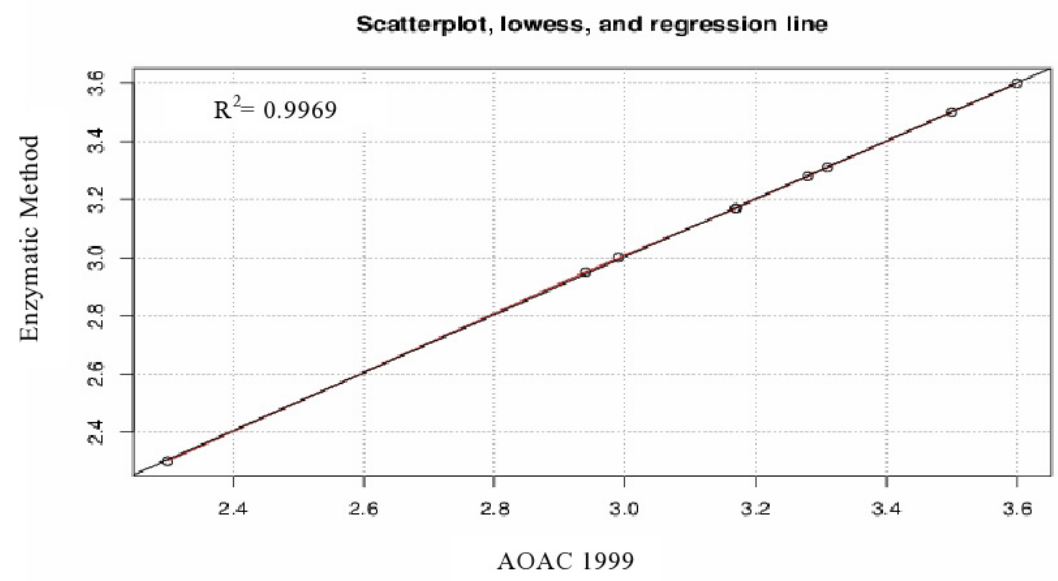

Figure 4. Simple linear regression analysis of the total oxalate between AOAC 1999 and enzymatic methods for herbs; the values used were the log transformation

Table 2. Effect of using different methods on the total oxalate concentration and precision of the method $(\mathrm{mg} / 100 \mathrm{~g})$

\begin{tabular}{lllll}
\hline Readings & Baker (1952) & Oke (1969) & Enzymatic (Kit) & AOAC 1999 \\
\hline $1^{\text {st }}$ reading & 668 & 750 & 921 & 905 \\
$2^{\text {nd }}$ reading & 650 & 700 & 914 & 900 \\
$3^{\text {rd }}$ reading & 550 & 740 & 916 & 895 \\
Average of total reading & 622.7 & 730 & 917 & 900 \\
$\pm *$ SEM & 36.70 & 26.45 & 2.1 & 5 \\
\hline
\end{tabular}

*standard error of the mean, each method was tested 9 times.

\subsection{Recovery Studies}

Known amounts of oxalate were added to 10 food samples tested before and known their amounts of oxalate, in addition to testing the reagent recoveries in order to obtain percentage of oxalate recovery. The recovery of added oxalate ranged from $93-107 \%$ for samples and $100.5 \%$ for reagent recoveries. 
The data represented in Table 3 shows the values of low concentration oxalate (less than $10 \mathrm{mg}$ oxalic acid / $100 \mathrm{~g}$ fresh weight), such as cabbage, cauliflower, courgette, cucumbers, garlic, onions (white), onions (spring- leaves only), peas (green) and turnip.

Table 3. Low oxalate concentration group (less than $10 \mathrm{mg}$ oxalic acid / 100g fresh weight)

\begin{tabular}{|c|c|c|c|c|}
\hline Vegetable/Taxonomic name & $\begin{array}{l}\text { Total oxalate } \\
\text { (Kit) }\end{array}$ & $\begin{array}{l}\text { *Soluble } \\
\text { oxalate } \\
\text { (Kit) }\end{array}$ & $\begin{array}{l}\text { Total oxalate } \\
\text { (AOAC 1999) }\end{array}$ & $\begin{array}{l}\text { *Soluble } \\
\text { oxalate } \\
\text { (AOAC 1999) }\end{array}$ \\
\hline \multicolumn{5}{|c|}{ **Log transformation values ( $\mathrm{mg} / 100 \mathrm{~g}$ fresh weight) } \\
\hline \multirow{2}{*}{ Cabbage (Brassica oleracea) } & $(0.60)^{* *}$ & ND & $(0.47)$ & ND \\
\hline & $4 \pm 0.15$ & & $3 \pm 0.06$ & \\
\hline \multirow{2}{*}{ Cauliflower (Brassica oleracea var. botrytis) } & $(0.65)$ & ND & $(0.69)$ & ND \\
\hline & $4.5 \pm 0.60$ & & $5 \pm 0.54$ & \\
\hline \multirow{2}{*}{ Courgette (Cucurbita pepo) } & $(0.77)$ & $(0.60)$ & $(0.90)$ & $(0.77)$ \\
\hline & $6 \pm 0.40$ & $4 \pm 0.09$ & $8 \pm 0.20$ & $6 \pm 0.52$ \\
\hline \multirow{2}{*}{ Cucumbers (Cucumis sativus) } & $(0.54)$ & $(0.30)$ & $(0.39)$ & $(0.17)$ \\
\hline & $3.5 \pm 0.20$ & $2 \pm 0.74$ & $2.5 \pm 0.07$ & $1.5 \pm 0.04$ \\
\hline \multirow{2}{*}{ Garlic (Allium sativum) } & $(0.77)$ & ND & $(0.84)$ & ND \\
\hline & $6 \pm 0.53$ & & $7 \pm 0.44$ & \\
\hline \multirow{2}{*}{ Onions (Allium cepa L.) (white) } & $(0.69)$ & $(0.54)$ & $(0.69)$ & $(0.47)$ \\
\hline & $5 \pm 0.34$ & $3.5 \pm 0.08$ & $5 \pm 0.40$ & $3 \pm 0.03$ \\
\hline \multirow{2}{*}{ Onions (Allium cepa) (spring- leaves only) } & $(0.84)$ & $(0.60)$ & $(0.77)$ & $(0.47)$ \\
\hline & $7 \pm 0.83$ & $4 \pm 0.02$ & $6 \pm 0.09$ & $3 \pm 0.05$ \\
\hline \multirow{2}{*}{ Peas (Pisum sativa) (green) } & $(0.84)$ & $(0.60)$ & $(0.90)$ & $(0.69)$ \\
\hline & $7 \pm 0.33$ & $4 \pm 0.67$ & $8 \pm 0.70$ & $5 \pm 0.56$ \\
\hline \multirow{2}{*}{ Turnip (Brassica rapa var. rapa) } & $(0.39)$ & $(0.30)$ & $(0.65)$ & $(0.47)$ \\
\hline & $2.5 \pm 0.07$ & $2 \pm 0.05$ & $4.5 \pm 0.18$ & $3 \pm 0.09$ \\
\hline
\end{tabular}

$*$ Insoluble oxalate $=$ total oxalate - soluble oxalate

** $\log$ transformation values

ND: not detected

The Table 4 shows moderate concentration group that contain 10-25 mg oxalic acid $/ 100 \mathrm{~g}$ fresh weight. This group include aubergine, bean (field), broccoli, corn, lettuce, peppers (green), peppers (red), tomatoes and watercress.

The data presented in Table 5 shows the vegetables, that contain $26-99 \mathrm{mg}$ oxalic acid $/ 100 \mathrm{~g}$ fresh weight. This group include beans, beans green, carrots, celery, mallow, okra, potatoes and sweet potatoes. 
Table 4. Moderate oxalate concentration group (10- $25 \mathrm{mg}$ oxalic acid /100 g fresh weight)

\begin{tabular}{|c|c|c|c|c|}
\hline Vegetable/Taxonomic name & $\begin{array}{l}\text { Total Oxalate } \\
\text { (Kit) }\end{array}$ & $\begin{array}{l}\text { Soluble } \\
\text { Oxalate } \\
\text { (Kit) }\end{array}$ & $\begin{array}{l}\text { Total Oxalate } \\
\text { (AOAC 1999) }\end{array}$ & $\begin{array}{l}\text { Soluble Oxalate } \\
\text { (AOAC 1999) }\end{array}$ \\
\hline \multicolumn{5}{|c|}{ Log transformation values ( $\mathrm{mg} / 100 \mathrm{~g}$ fresh weight) } \\
\hline \multirow{2}{*}{ Aubergine (Solanum melongena) } & $(1.19)$ & $(0.95)$ & $(1.20)$ & $(0.84)$ \\
\hline & $15.5 \pm 0.95$ & $9 \pm 0.66$ & $16 \pm 0.85$ & $7 \pm 0.87$ \\
\hline \multirow{2}{*}{$\begin{array}{l}\text { Bean (Field) (Phaseolus aureus) } \\
\text { (seeds only) }\end{array}$} & $(1.07)$ & $(0.60)$ & $(1.0)$ & $(0.47)$ \\
\hline & $12 \pm 0.83$ & $4 \pm 0.41$ & $10 \pm 0.63$ & $3 \pm 0.32$ \\
\hline \multirow{2}{*}{ 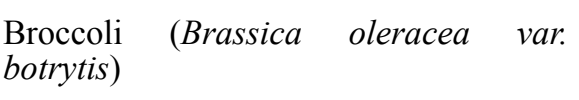 } & $(1.30)$ & $(0.84)$ & $(1.30)$ & $(0.69)$ \\
\hline & $20 \pm 0.85$ & $7 \pm 53$ & $20 \pm 1.3$ & $5 \pm 0.32$ \\
\hline \multirow{2}{*}{$\begin{array}{l}\text { Corn, (Zea mays subsp. mays) (corn } \\
\text { seeds) }\end{array}$} & $(1.09)$ & $(0.77)$ & $(1.07)$ & $(0.60)$ \\
\hline & $12.5 \pm 0.23$ & $6 \pm 54$ & $12 \pm 0.65$ & $4 \pm 0.19$ \\
\hline \multirow{2}{*}{ Lettuce (Lactuca sativa L.) } & $(1.07)$ & $(0.69)$ & $(1.0)$ & $(0.47)$ \\
\hline & $12 \pm 0.76$ & $5 \pm .08$ & $10 \pm 0.70$ & $3 \pm 0.35$ \\
\hline \multirow{2}{*}{$\begin{array}{l}\text { Peppers (green) Capsicum annum var. } \\
\text { grossum }\end{array}$} & $(1.0)$ & $(0.84)$ & $(1.04)$ & $(0.77)$ \\
\hline & $10 \pm 034$ & $7 \pm 0.33$ & $11 \pm 0.43$ & $6 \pm 0.21$ \\
\hline \multirow{2}{*}{$\begin{array}{l}\text { Peppers (red) Capsicum annum var. } \\
\text { grossum }\end{array}$} & $(1.04)$ & $(0.77)$ & $(1.0)$ & $(0.60)$ \\
\hline & $11 \pm 0.82$ & $6 \pm .05$ & $10 \pm 0.6$ & $4 \pm 0.01$ \\
\hline \multirow{2}{*}{ Tomatoes (Lycopersicon esculentum) } & $(1.27)$ & $(1.20)$ & $(1.11)$ & $(1.07)$ \\
\hline & $19 \pm 0.38$ & $16 \pm 0.89$ & $13 \pm 0.49$ & $12 \pm 0.74$ \\
\hline \multirow{2}{*}{ Watercress (Nasturium oficinale) } & $(1.14)$ & $(0.90)$ & $(1.17)$ & $(0.77)$ \\
\hline & $14 \pm 0.76$ & $8 \pm 0.43$ & $15 \pm 0.74$ & $6 \pm 0.54$ \\
\hline
\end{tabular}

Table 5. High oxalate concentration (26- $99 \mathrm{mg}$ oxalic acid /10g fresh weight)

\begin{tabular}{|c|c|c|c|c|}
\hline Vegetable/Taxonomic name & $\begin{array}{l}\text { Total oxalate } \\
\text { (Kit) }\end{array}$ & $\begin{array}{l}\text { Soluble } \\
\text { oxalate } \\
\text { (Kit) }\end{array}$ & $\begin{array}{l}\text { Total oxalate } \\
\text { (AOAC 1999) }\end{array}$ & $\begin{array}{l}\text { Soluble } \\
\text { oxalate } \\
\text { (AOAC 1999) }\end{array}$ \\
\hline \multicolumn{5}{|c|}{ Log transformation values ( $\mathrm{mg} / 100 \mathrm{~g}$ fresh weight) } \\
\hline \multirow{2}{*}{ Beans (Vicia faba) } & $(1.75)$ & $(1.64)$ & $(1.74)$ & $(1.60)$ \\
\hline & $57 \pm 2.5$ & $44 \pm 1.8$ & $55 \pm 2.7$ & $40 \pm 1.6$ \\
\hline \multirow{2}{*}{ Beans, green (Phaseolus vulgaris) } & $(1.60)$ & $(1.49)$ & $(1.54)$ & $(1.38)$ \\
\hline & $40 \pm 1.5$ & $31 \pm 0.92$ & $35 \pm 0.78$ & $24 \pm 0.66$ \\
\hline \multirow{2}{*}{ Carrots (Daucas carota) } & $(1.61)$ & $(1.55)$ & $(1.57)$ & $(1.50)$ \\
\hline & $41 \pm 2.01$ & $36 \pm 0.76$ & $38 \pm 2.3$ & $32 \pm 2.7$ \\
\hline \multirow{2}{*}{ Celery (Apium graveolens var. dulce) } & $(1.43)$ & $(0.77)$ & $(1.30)$ & $(0.69)$ \\
\hline & $27 \pm 1.58$ & $6 \pm 0.54$ & $20 \pm 0.87$ & $5 \pm 0.21$ \\
\hline \multirow{2}{*}{ Mallow (Malva sylvestris) } & $(1.74)$ & $(1.68)$ & $(1.74)$ & $(1.69)$ \\
\hline & $56 \pm 1.10$ & $48 \pm 1.89$ & $55 \pm 1.66$ & $50 \pm 1.57$ \\
\hline \multirow{2}{*}{ Okra (Abelmoschus esculentus) } & $(1.65)$ & $(1.65)$ & $(1.61)$ & $(1.61)$ \\
\hline & $45 \pm 0.56$ & $45 \pm 0.22$ & $41 \pm 0.40$ & $41 \pm 0.40$ \\
\hline \multirow{2}{*}{ Potatoes (Solanum tuberosum L.) } & $(1.43)$ & $(1.32)$ & $(1.47)$ & $(1.32)$ \\
\hline & $27 \pm 0.08$ & $21 \pm 0.04$ & $30 \pm 0.56$ & $21 \pm 0.32$ \\
\hline \multirow{2}{*}{ Sweet potatoes (Ipomoea batatas) } & $(1.68)$ & $(1.57)$ & $(1.71)$ & $(1.54)$ \\
\hline & $48 \pm 0.13$ & $38 \pm 0.04$ & $52 \pm 1.20$ & $35 \pm 1.09$ \\
\hline
\end{tabular}

Fw: fresh weight; insoluble oxalate such as calcium oxalate can be calculated from subtraction total oxalate from soluble oxalate 
In addition to this groups some leafy green vegetables showed in Table 6 an oxalic content over 99-900 mg oxalic $\mathrm{acid} / 100 \mathrm{~g}$ fresh weight. This oxalic acid content can be considered as very rich concentration, this group include Swiss chard, molokhia, parsley, purslane, spinach and vine leaves (fresh).

Table 6. Very high oxalate concentration (more than 99-900 mg oxalic acid / 100g fresh weight)

\begin{tabular}{|c|c|c|c|c|}
\hline Vegetable/Taxonomic name & $\begin{array}{l}\text { Total Oxalate } \\
\text { (Kit) }\end{array}$ & $\begin{array}{l}\text { Soluble } \\
\text { Oxalate } \\
\text { (Kit) }\end{array}$ & $\begin{array}{l}\text { Total Oxalate } \\
\text { (AOAC 1999) }\end{array}$ & $\begin{array}{l}\text { Soluble Oxalate } \\
\text { (AOAC 1999) }\end{array}$ \\
\hline \multicolumn{5}{|c|}{ Log transformation values ( $\mathrm{mg} / \mathrm{l} 00 \mathrm{~g}$ fresh weight) } \\
\hline Swiss chard & $(2.90)$ & $(2.77)$ & $(2.90)$ & $(2.75)$ \\
\hline (Beta vulgaris subsp. cicla) & $812 \pm 3.54$ & $600 \pm 40.87$ & $800 \pm 4.76$ & $570 \pm 3.56$ \\
\hline \multirow{2}{*}{$\begin{array}{l}\text { Molokhia (Corchorus olitorius L) } \\
\text { (leaves mainly) }\end{array}$} & $(2.65)$ & $(2.14)$ & $(2.65)$ & $(2.11)$ \\
\hline & $457 \pm 3.60$ & $140 \pm 0.85$ & $450 \pm 2.50$ & $130 \pm 1.09$ \\
\hline \multirow{2}{*}{ Parsley (Petroselinum crispum) } & $(2.19)$ & $(1.51)$ & $(2.17)$ & $(1.47)$ \\
\hline & $156 \pm 1.02$ & $33 \pm 0.76$ & $150 \pm 1.30$ & $30 \pm 0.70$ \\
\hline \multirow{2}{*}{ Purslane (Portulaca oleracea) } & (2.93) & $(2.74)$ & $(2.92)$ & $(2.74)$ \\
\hline & $862 \pm 3.10$ & $559 \pm 1.46$ & $850 \pm 7.31$ & $550 \pm 3.10$ \\
\hline \multirow{2}{*}{ Spinach (Spinacia oleracea) } & $(2.96)$ & $(2.89)$ & $(2.95)$ & $(2.88)$ \\
\hline & $917 \pm 2.1$ & $785 \pm 1.59$ & $900 \pm 50$ & $770 \pm 4.20$ \\
\hline \multirow{2}{*}{ Vine leaves (fresh) } & $(2.48)$ & (2.30) & $(2.46)$ & $(2.26)$ \\
\hline & $302 \pm 2.01$ & $202 \pm 1.09$ & $290 \pm 2.10$ & $185 \pm 1.70$ \\
\hline
\end{tabular}

The data presented in Table 7. shows the concentration of oxalate $(\mathrm{mg} / 100 \mathrm{~g})$ in a selected Egyptian grown fruits. This group include apples, apricots fresh, guava, strawberries and mangoes.

Table 7. Concentration of oxalate $(\mathrm{mg} / 100 \mathrm{~g})$ in a selected Egyptian grown fruits

\begin{tabular}{|c|c|c|c|c|}
\hline Fruit/Taxonomic name & $\begin{array}{l}\text { Total oxalate } \\
\text { (Kit) }\end{array}$ & $\begin{array}{l}\text { Soluble oxalate } \\
\text { (Kit) }\end{array}$ & $\begin{array}{l}\text { Total oxala } \\
\text { (AOAC 1999) }\end{array}$ & $\begin{array}{l}\text { Soluble oxalate } \\
\text { (AOAC 1999) }\end{array}$ \\
\hline \multicolumn{5}{|c|}{ Log transformation values ( $\mathrm{mg} / 100 \mathrm{~g}$ fresh weight) } \\
\hline \multirow{2}{*}{ Apples (Malus domestica) } & $(1.04)$ & $(0.77)$ & $(0.95)$ & $(0.60)$ \\
\hline & $11 \pm 0.9$ & $6 \pm 0.32$ & $9 \pm 0.75$ & $4 \pm 0.21$ \\
\hline \multirow{2}{*}{ Apricots (Prunus armeniaca) (fresh) } & $(1.68)$ & $(1.61)$ & (1.69) & $(1.64)$ \\
\hline & $48 \pm 80$ & $41 \pm 11$ & $50 \pm 15$ & $44 \pm 50$ \\
\hline \multirow{2}{*}{ Guava (Psidium guajava $\mathrm{L}$ ) } & $(1.25)$ & $(1.25)$ & (1.23) & $(1.14)$ \\
\hline & $18 \pm 20$ & $18 \pm 20$ & $17 \pm 1.5$ & $14 \pm 1.7$ \\
\hline \multirow{2}{*}{ Strawberries (Fragaria ananassa Duch) } & $(1.39)$ & $(1.27)$ & (1.36) & $(1.30)$ \\
\hline & $25 \pm 4$ & $19 \pm 1.3$ & $23 \pm 1.9$ & $20 \pm 5$ \\
\hline \multirow{2}{*}{ Mangoes (Mangifera indica) } & $(1.07)$ & $(0.84)$ & (1.0) & $(0.90)$ \\
\hline & $12 \pm 30$ & $7 \pm 0.70$ & $10 \pm 10$ & $8 \pm 2.4$ \\
\hline
\end{tabular}

The data in Table 8. Showed the oxalate concentration of some common herbs (mg/100 g D.W.) of daily selected common herbs that include caraway seed, cardamom (green), cinnamon, coriander seeds, cumin, curry powder, ginger, nutmeg and turmeric powder. 
Table 8. Oxalate concentration of some common herbs (mg/100g D.W.) of daily selected common herbs

\begin{tabular}{lllll}
\hline Herb/Taxonomic name & $\begin{array}{l}\text { Total oxalates } \\
(\text { Kit })\end{array}$ & $\begin{array}{l}\text { Soluble oxalates } \\
(\text { Kit })\end{array}$ & $\begin{array}{l}\text { Total oxalate } \\
(\text { AOAC 1999) }\end{array}$ & $\begin{array}{l}\text { Soluble oxalate } \\
\text { (AOAC 1999) }\end{array}$ \\
\hline & Log transformation & values $($ mg/100g dry & weight) & \\
\hline Caraway seeds (Carum carvi) & $(2.95)$ & $(1.77)$ & $(2.94)$ & $(1.76)$ \\
& $900 \pm 55$ & $60 \pm 21$ & $890 \pm 78$ & $58 \pm 9$ \\
Cardamom (green) & $(3.60)$ & $(3.59)$ & $(3.60)$ & $(3.59)$ \\
(Elettaria cardamomum) & $4014 \pm 102$ & $3977 \pm 123$ & $4000 \pm 115$ & $3970 \pm 135$ \\
Cinnamon & $(3.50)$ & $\mathrm{ND}$ & $(3.50)$ & $\mathrm{ND}$ \\
(Cinnamomum zelanicum) & $3200 \pm 117$ & & $3195 \pm 146$ & \\
Coriander seeds & $(3.00)$ & $\mathrm{ND}$ & $(2.99)$ & $\mathrm{ND}$ \\
(Coriandrum sativum) & $1005 \pm 150$ & & $995 \pm 56$ & \\
Cumin (Cuminum cyminum) & $(3.17)$ & $(2.05)$ & $(3.17)$ & $(2.02)$ \\
& $1505 \pm 150$ & $114 \pm 11$ & $1500 \pm 147$ & $105 \pm 10$ \\
Curry powder & $(3.31)$ & $(1.81)$ & $(3.31)$ & $(1.88)$ \\
(Murraya koenigii) & $1070 \pm 126$ & $65 \pm 80$ & $1065 \pm 96$ & $77 \pm 12$ \\
Ginger (Zingiber officinale) & $(3.17)$ & $(3.14)$ & $(3.17)$ & $(3.13)$ \\
& $1488 \pm 88$ & $1390 \pm 57$ & $1480 \pm 81$ & $1379 \pm 60$ \\
Nutmeg (Myristica fragrans) & $(2.30)$ & $(1.66)$ & $(2.30)$ & $(1.70)$ \\
& $201 \pm 11$ & $46 \pm 6$ & $200 \pm 32$ & $51 \pm 8$ \\
Turmeric powder & $(3.28)$ & $(3.25)$ & $(3.28)$ & $(3.25)$ \\
(Curcuma domestica) & $1910 \pm 68$ & $1812 \pm 85$ & $1914 \pm 67$ & $1809 \pm 79$ \\
\hline & & & & \\
\hline
\end{tabular}

\section{Discussion}

Since absorbed dietary oxalate can make a significant contribution to urinary oxalate levels therefore the Egyptian consumers and dieticians always rely on oxalate content of food but from oxalate analyzers that are different circumstances because the unavailability of a local database. Consequently, the aim of this work was to establish a local database of oxalate included the common foods in Egypt such as fül, molokhia, baladi corn, vine leaves, mallows, mangoes and guava. The total food analysed in this study were 37 fruits and vegetables and 9 daily common herbs. The AOAC 1999 and Trinity biotech kit for oxalate in urine were used to evaluate the total and soluble oxalate.

Advantages of selected methods; AOAC 1999 and enzymatic kit, were that they were time efficient and had relatively fewer steps. Results indicate that oxalate kit accuracy and precision was higher than AOAC and given higher values of oxalate than AOAC 1999. Enzymatic method is adequate to the analysis of oxalate contents in the examined foods, and the inter-day precision of the method expressed as standard error of mean was good (2.1), with an accuracy of the recovery of added oxalate ranged from $93-107 \%$ for samples and $100.5 \%$ for reagent recoveries.

In Egypt, consumption of vegetables is an important part of food habits, therefore grown vegetables were screened for total and water soluble oxalate that were divided into four groups:

(i) Low oxalate concentration vegetables that were less than $10 \mathrm{mg} / 100 \mathrm{~g}$ F.W., include cabbage, cauliflower, courgette, cucumbers, garlic, onions, spring onions, peas and turnip, which are the most vegetables consumed in Egypt and would have a beneficial effect on patients with calcium oxalate stones.

(ii) Moderate oxalate concentration vegetables that contained 10-25 mg/100 g F.W. include aubergine, field bean, broccoli, baladi corn, lettuce, peppers (green and red), tomatoes, and watercress.

(iii) High oxalate concentration vegetables that contained 26- 99 mg/100 g F.W. include beans, green beans, carrots, celery, mallow, okra, potatoes, and sweet potatoes.

(iv) Very high oxalate concentration vegetables that contained 100- $900 \mathrm{mg} / 100 \mathrm{~g}$ F.W. oxalic acid include Swiss chard, molokhia (leaves mainly), parsley, purslane, spinach, vine leaves (fresh). 
Some fruits (apples, apricots (fresh), guava, strawberries and mangoes) were also screened for total and water soluble oxalate. Apples in this study were found to have higher amounts than previously reported studies with total oxalate levels between 0-2 mg/100 g (Holmes \& Kennedy, 2000; Hönow \& Hesse, 2002). In the study conducted by El-Wahsh et al. (2012) a range of 1.3-2.6 mg total oxalate/100 $\mathrm{g}$ for apples were found.

Several studies were conflicting with regard to the reported oxalate level of strawberries with total oxalate levels ranging from $2.9 \mathrm{mg} / 100 \mathrm{~g}$ (Hönow \& Hesse, 2002) to $23.4 \mathrm{mg} / 100 \mathrm{~g}$ (Ogawa, Takahashi, \& Kitagawa, 1984). In El-Wahsh et al., 2012, strawberries were reported to contain $6.0 \mathrm{mg}$ and $2.5 \mathrm{mg}$ of total and soluble oxalate $/ 100 \mathrm{~g}$, respectively. In the present study, amount of total oxalate ranged from $23-25 \mathrm{mg} / 100 \mathrm{~g}$ F.W. The variation in oxalate values in different sources of plants can be affected by factors such as soil quality, climate or different state of fruit ripeness (Libert \& Franceschi, 1987). In addition, differences could also be due to dissimilarity in preparation of the samples and analytical techniques.

In Egypt, sweet potato is a common food eaten as a dessert while potatoes are staple food served as a main dish. The oxalate content of sweet potato and potatoes were found to be 48 and $27 \mathrm{mg} / 100 \mathrm{~g}$ F.W., respectively, this is similar to the results of a Taiwanese study $(48.6 \mathrm{mg} / 100 \mathrm{~g}$.) (Tsai et al., 2005).

fül (Vicia faba) is a staple food in Egypt, especially for low income people, with the amount of soluble oxalate ranges between $40-44 \mathrm{mg} / 100 \mathrm{~g} \mathrm{D}$.W. (total oxalate $55-57 \mathrm{mg} / 100 \mathrm{~g} \mathrm{D} . \mathrm{W}$.) there is a possibility that through soaking and cooking oxalates are lost, but care should be paid to the spices that are added to the ful, specifically cumin which has mega amounts of oxalate that found to be (1505-1500 mg/100g D.W.) .

Molokhia is a leafy green vegetable, another traditional food in Egypt, served as a thick green soup beside the main dish that is usually a meat source. Molokhia belongs to very high oxalate concentration group (450-457 mg/100 g F.W.) about $31 \%$ of it have water soluble oxalate, coriander seed is used to spice traditional molokhia that is found to contain $995-1005 \mathrm{mg} / 100 \mathrm{~g}$ D.M. Spinach and mallow are traditional vegetables in Egypt that are eaten in winter similarly to molokhia in terms of adding spices. Mangoes are summer fruit in Egypt that are eaten as whole or as juices and contains from $8-12 \mathrm{mg} / 100 \mathrm{~g}$ fresh weights.

Oxalate concentration of some common herbs (mg/100 g D.W.) of a selected common herbs include green cardamom, coriander seeds, caraway seeds, cinnamon, cumin, curry powder, ginger, nutmeg and turmeric powder that also important values for Egyptian cuisine. The data presented in Table 8. Indicates to the concentration of oxalate in some common herbs used in the Egyptian cuisine.

Noticeably most these herbs contain extensive amounts of oxalate that exceeds $1000 \mathrm{mg} / 100 \mathrm{~g}$ dry weight that may consumers, patients and dieticians focus on high and very high concentrations of vegetables apart from herbs that added to food. High consumption of oxalate rich foods can lead to secondary hyperoxaluria, a major risk factor for calcium oxalate stone formation, and even acute renal failure in the case of excessive dietary oxalate intake (Simpson et al., 1999).

About $75 \%$ of all kidney stones are composed primarily of calcium oxalate (Williams \& Wandzilak, 1989) and hyperoxaluria is a primary risk factor for this disorder (Goldfarb, 1988; Robertson \& Hughes, 1993). Urinary oxalate originates from a combination of absorbed dietary oxalate and endogenous formation from oxalate precursors such as ascorbic acid and glyoxylate (Williams \& Wandzilak, 1989). Restriction of dietary oxalate intake has been proposed to prevent the formation of calcium oxalate kidney stones.

\section{Conclusion}

This study analysed traditional foods to establish a local database of oxalate content in 37 Egyptian grown fruits and vegetables and 9 selected daily common herbs. Two methods were used for screening the Egyptian foods for oxalate concentration. This study was analysed the traditional foods in Egypt such as fūl, molokhia, baladi corn, vine leaves, mallows, mangoes and guava. Total oxalate varied greatly among the vegetables examined, ranged from 4 to 917 (mg/100 g F.W). Total oxalate of analysed fruits ranged from 9 to 50 (mg/100 g F. W). Extensive amounts of total oxalate were found in daily common herbs $201-4014$ (mg/100 g D.W.). We hope the data obtained herein can help dieticians, patients and urologists to instruct the public on urolithiasis prevention.

\section{References}

Al-Wahsh, I. A., Wu, Y., \& Liebman, M. (2012). A Comparison of Two Extraction Methods for Food Oxalate Assessment. Journal of Food Research, 1(2), 233-239. http://dx.doi.org/10.5539/jfr.v1n2p233

AOAC. (1999). Association of Official Analytical Chemists, Official Methods of Analysis. Washington DC.

Baker, C. J. L. (1952). The determination of oxalates in fresh plant material. Analyst, 77, 340. http://dx.doi.org/10.1039/an9527700340 
Bataille, P., \& Fournier, A. (2001). Calcium supply in calcium lithiasis. Journal of Medical Nutrition, 37, 9-12.

Eheart, J. F., \& Massey, Jr. P. H. (1962). Factors affecting the oxalate content in spinach. Chemical Constituents of Plants, 10(4), 325.

Franceschi, V. R., \& Horner, H. T. Jr. (1980). Calcium oxalate crystals in plants. The Botanical Review, 46, 361-427. http://dx.doi.org/10.1007/BF02860532

Goldfarb, S. (1988). Dietary factors in the pathogenesis and prophylaxis of calcium nephrolithiasis. Kidney International, 34, 544-555. http://dx.doi.org/10.1038/ki.1988.216

Hodgkinson, A. (1977). Oxalic acid metabolism in higher plants. In A. Hodgkinson (Ed.), Oxalic Acid Biology and Medicine (pp. 131-158). New York: Academic Press.

Holmes, R. P., \& Assimos, D. G. (1998). Glyoxylate synthesis, and its modulation and influence on oxalate synthesis. Journal of Urology, 160, 1617-1624. http://dx.doi.org/10.1097/00005392-199811000-00003

Holmes, R. P., \& Kennedy, M. (2000). Estimation of the oxalate content of foods and daily oxalate intake. Kidney International, 57, 1662-1667. http://dx.doi.org/10.1046/j.1523-1755.2000.00010.x

Hönow, R., \& Hesse, A. (2002). Comparison of extraction methods for the determination of soluble and total oxalate in foods by HPLC-enzyme-reactor. Food Chemistry, 78(4), 511-521. http://dx.doi.org/10.1016/S0308-8146(02)00212-1

Keates, S. A., Tarlyn, N., Loewus, F. A., \& Franceschi, V. R. (2000). 1-Ascorbic acid and 1-galactose are sources of oxalic acid and calcium oxalate in Pistia stratiotes. Phytochemistry, 53, 433-440. http://dx.doi.org/10.1016/S0031-9422(99)00448-3

Li, X. X., \& Franceschi, V. R. (1990). Distribution of peroxisomes and glycolate metabolism in relation to calcium oxalate formation in Lemna minor L. European Journal of Cell Biology, 51, 9-16.

Libert, B., \& Franceschi, V. R. (1987). Oxalate in crop plants. Journal of Agricultural and Food Chemistry, 35(6), 926-938. http://dx.doi.org/10.1021/jf00078a019

Liebman, M. (2002). The Truth About Oxalate Answers to Frequently Asked Questions. The Vulvar Pain Newsletter.

Massey, L. K., Roman-Smith, H., \& Sutton R. A. L. (1993). Effect of dietary oxalate and calcium on urinary oxalate and risk of formation of calcium oxalate kidney stones. Journal of American Diet Association, 93, 901-906. http://dx.doi.org/10.1016/0002-8223(93)91530-4

Nakata, P. A., \& McConn, M. M. (2000). Isolation of Medicago truncatula Mutants Defective in Calcium Oxalate Crystal Formation. Plant Physiology, 124, 1097-1104. http://dx.doi.org/10.1104/pp.124.3.1097

Nakagawa, Y., Shimazu, K., Ebihara, M., \& Nakagawa, K. (1999). Aspergillus niger pneumonia with fatal pulmonary oxalosis. Journal of Infectious Chemotherapy, 5, 97-100. http://dx.doi.org/10.1007/s101560050016

Noonan, S. C., \& Savage, G. B. (1999). Oxalate content of foods and its effect on humans. Asia pacific Journal of Clinical Nutrition, 8(1), 64-74. http://dx.doi.org/10.1046/j.1440-6047.1999.00038.x

Nuss, R. F., \& Loewus, F. A. (1978). Further studies on oxalic acid biosynthesis in oxalate- accumulating plants. Plant Physiology, 61, 590-592. http://dx.doi.org/10.1104/pp.61.4.590

Ogawa, Y., Takahashi, S., \& Kitagawa, R. (1984). Oxalate content in common Japanese foods. Acta Urologica Japonica, 30, 305-310.

Oke, Z. O. L. (1969). Oxalic acid and plants and in nutrition. World Review of Nutrition and Dietetics, 10, 262-303.

Palaniswamy, U. R., Bible, B. B., \& McAvoy, R. J. (2004). Oxalic acid concentrations in purslane (Portulaca oleraceae L.) is altered by the stage of harvest and the nitrate to ammonium ratios in hydroponics. Scientia Horticulturae, 102, 267-275. http://dx.doi.org/10.1016/j.scienta.2004.01.006

Quinteros, A., Farré, R., \& Lagarda, M. J. (2003). Effect of cooking on oxalate content of pulses using an enzymatic procedure. International Journal of Food Science and Nutrition, 54(5), 373-377. http://dx.doi.org/10.1080/09637480310001595270

Rahman, M. M., Yamamoto, M., Niimi, M., \& Kawamura, O. (2008a). Effect of nitrogen fertilization on oxalate content in Rhodesgrass, Guineagrass and Sudangrass. Asian-Austrilian Journal of Animal Science, 21(2), 214-219. 
Robertson, W. G., \& Hughes, H. (1993). Importance of mild hyperoxaluria in the pathogenesis of urolithiasis-new evidence from studies in the Arabian peninsula. Scanning Microscopy, 7, 391-401.

Simpson, T. S., Savage, G. P., Sherlock, R., Vanhanen, L. P. (1999). Oxalate content of silver beet leaves (Beta vulgaris var. cicla) at different stages of maturation and the effect of cooking with different milk sources. Journal of Agricultural and Food Chemistry, 57(22), 10804-10808. http://dx.doi.org/10.1021/jf902124w

Tsai, J. Y., Huang, J. K, Wu, T. T., \& Lee, Y. H. (2005). Comparison of Oxalate Content in Foods and Beverages in Taiwan. JTUA, 16, 93-98.

USDA. (1984). Oxalic acid content of selected vegetables. In: Agriculture handbook, No. 8-11, Vegetables and vegetable products. 15 Jan. 2008. http://www.nal.usda.gov/fnic/foodcomp/Data/Other/oxalic.html.

Wessa, P. (2012). Linear Regression Graphical Model Validation (v1.0.7) in Free Statistics Software (v1.1.23-r7), Office for Research Development and Education. Retrieved from http://www.wessa.net/rwasp_linear_regression.wasp/

Williams, H. E., \& Wandzilak, T. R. (1989). Oxalate synthesis, transport and the hyperoxaluricsyndromes. Journal of Urology, 141, 742-747.

Yang, J., \& Loewus, F. A. (1975). Metabolic conversion of L-ascorbic acid in oxalate-accumulating plants. Plant Physiology, 56, 283-285. http://dx.doi.org/10.1104/pp.56.2.283

\section{Copyrights}

Copyright for this article is retained by the author(s), with first publication rights granted to the journal.

This is an open-access article distributed under the terms and conditions of the Creative Commons Attribution license (http://creativecommons.org/licenses/by/3.0/). 